\title{
Aspherical magnetically modulated optical nanoprobes (MagMOONs)
}

\author{
Jeffrey N. Anker, Caleb Behrend, and Raoul Kopelman ${ }^{\mathrm{a})}$ \\ Chemistry Department, The University of Michigan Ann Arbor, Michigan 48109-1055
}

(Presented on 12 November 2002)

\begin{abstract}
Aspherical magnetic particles orient in a magnetic field due to magnetic shape anisotropy. They also emit different fluxes of light from their different geometric faces due to self-absorption and total internal reflection within the particles. The particles rotate in response to rotating magnetic fields and appear to blink as they rotate. We have made pancake and chain shaped particles and magnetically modulated their fluorescent intensities. Demodulating the signal extracts the probe fluorescence from electronic and optical backgrounds dramatically increasing signal to noise ratios. The probes have applications in sensitive and rapid immunoassays, improved intracellular sensors, and inexpensive single molecule analysis. (ㅇ 2003 American Institute of Physics.
\end{abstract}

[DOI: $10.1063 / 1.1556926]$

\section{INTRODUCTION}

Fluorescence is the most sensitive method available for molecular detection and chemical imaging. It is even used for real time imaging of single molecules at ambient conditions with high spatial and spectral resolution. ${ }^{1,2}$ Fluorescent dyes are commonly used to: study intracellular chemical concentration changes, ${ }^{3,4}$ measure immunochemical concentrations in fluids,${ }^{5}$ tag molecules on cell surfaces or tissues, ${ }^{6}$ and research protein folding. ${ }^{7,8}$ They are critical to understanding how cells function, for rapid drug discovery and for detecting minute quantities of pathogens or DNA. Nevertheless, background fluorescence from sample and instrument optics makes detecting low levels of fluorescence or small changes in fluorescence challenging. Rejecting background fluorescence and increasing signal to noise ratios by orders of magnitude would thus lead to important advances in molecular and biomedical sciences.

Blinking or flashing light sources are easily distinguished from light sources that radiate continuously or that slowly change their intensity. It is an established scientific practice to modulate signals in order to increase signal to noise ratios. We have developed aspherical magnetically modulated optical nanoprobes (MagMOONs) that blink in response to rotating magnetic fields. The probes orient in external fields due to magnetic shape anisotropy. They also absorb and emit different fluxes of light in different orientations as a result of total internal reflection and self-absorption within the particles (analogous to Venetian blinds), see Fig. 1. Separating the blinking probe signal from the unmodulated background allows simple and sensitive detection of low analyte concentrations even in the presence of autofluorescence and other backgrounds. Thus MagMOONs can ex-

a) Author to whom correspondence should be addressed; electronic mail: Kopelman@umich.edu tend the range of dyes that may be used for sensing and also extend the range of conditions under which experiments will work. Compared with physical separation of magnetic particles using magnetic field gradients, ${ }^{9}$ the modulation of optical properties reduces sample preparation time as well as instrument complexity. Importantly, the MagMOON rotation rate is independent of particle size assuming constant viscosity and magnetic susceptibility. ${ }^{10}$

In this paper we focus on forming and using aspherical MagMOONs. Metal-capped permanent magnetic particles give another type of MagMOON. ${ }^{11}$ Metal-capped MagMOONs are permanent magnet microspheres and nanospheres that are coated on one hemisphere with vapor deposited metal. The particle is then magnetized so that the uncoated light-emitting side is the magnetic north of the particle. The two MagMOON types can be distinguished, enabling signal multiplexing, because only $90^{\circ}$ separates the aspherical MagMOONs" "on" and "off" states as opposed to $180^{\circ}$ for metal-capped MagMOONs. Probes that respond
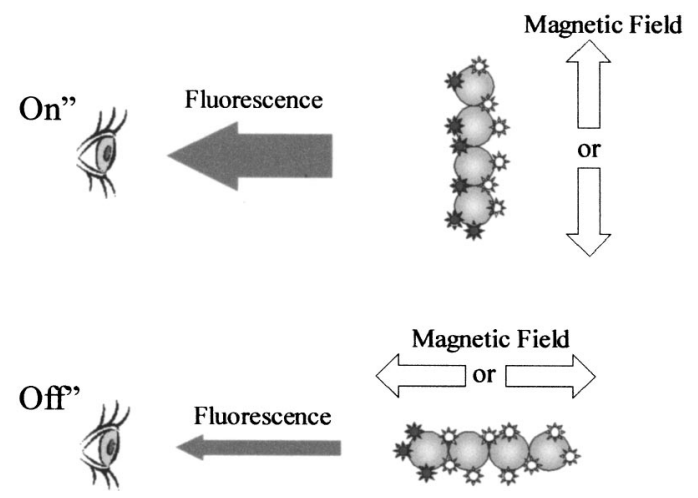

FIG. 1. An external magnetic field orients a chain shaped aspherical MagMOON causing its fluorescent excitation and observed emission to blink on and off as it rotates. 


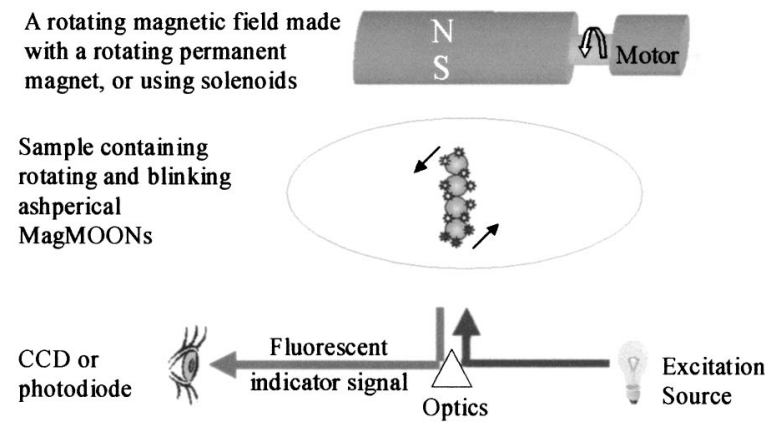

FIG. 2. A setup to magnetically modulate MagMOONs and separate their blinking signal from the unmodulated background.

to modulated magnetic field gradients instead of field orientations give yet another distinguishable type of MagMOON.

Figure 2 illustrates a setup to magnetically modulate MagMOONs and separate their blinking signal from unmodulated backgrounds. A sample containing aspherical MagMOONs and interfering backgrounds is viewed with an Olympus IMT2 epifluorescent microscope. A program written in Labview (National Instruments, Austin, TX) controls a stepper motor interfaced to a computer parallel port. The motor rotates a magnet clockwise and counterclockwise $90^{\circ}$ in order to orient MagMOONs on and off. Spectra are acquired and saved after every rotation. The probe spectrum is calculated as the average "on minus off" spectrum. Statistical analysis of the time series of "on minus off" spectra using principal components analysis can be used to further reduce interference from background signals that fluctuate independently from the MagMOONs.

\section{CHAIN SHAPED MagMOONs}

Chains of magnetic particles form spontaneously in magnetic fields. If the particles are superparamagnetic then the chains dissipate when the field is removed. Selfabsorption and scattering due to large concentrations of iron oxide within the particles causes the fluorescence intensity to depend on the orientation of the chains. Figure 3 shows images and spectra of $1-2-\mu \mathrm{m}$-diam fluorescent polystyrene microspheres (Polysciences) that form into chains in an external field. Comparing the original $\mathrm{ON}$ and $\mathrm{OFF}$ spectra [3(c)] to the ON minus OFF blinking signal [3(d)], subtrac-
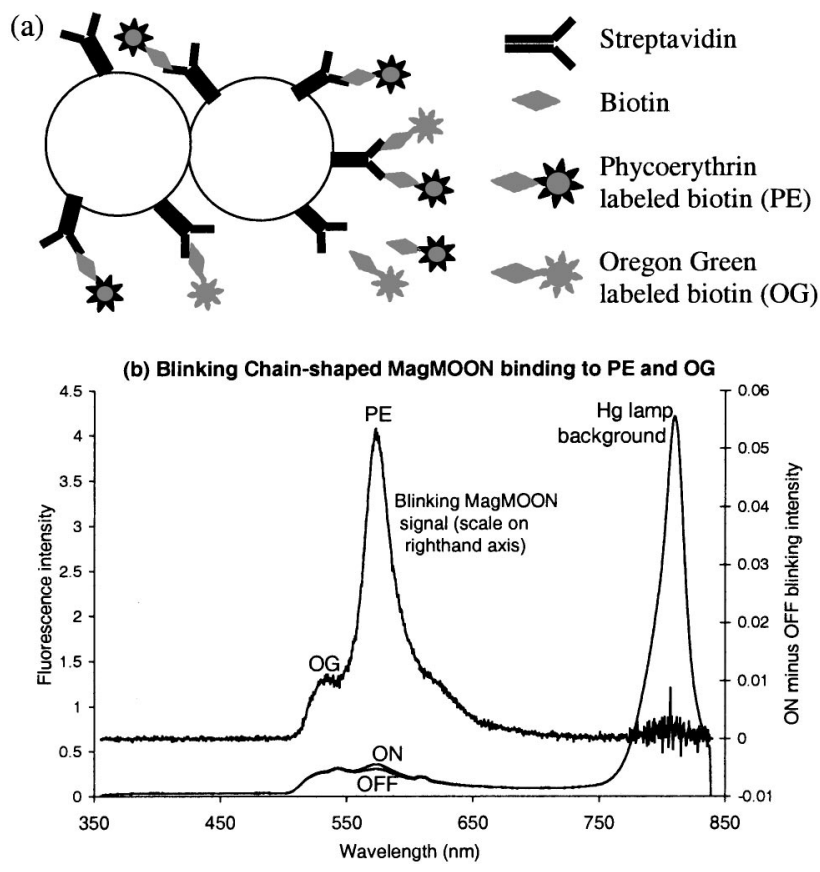

FIG. 4. Chain shaped streptavidin-coated MagMOONs detect relative concentrations of Oregon Green labeled biotin (OG) and phycoerythrin labeled biotin (PE) even in the presence of background. (a) Assay schematic. (b) MagMOON spectrum is separated from background spectrum.

tion attenuates by over three orders of magnitude the mercury lamp background interference peak at $800 \mathrm{~nm}$.

To demonstrate the principle of MagMOON immunoassays (Fig. 4), streptavidin superparamagnetic nanospheres $870 \mathrm{~nm}$ in diameter (Bangs Labs) were immersed in solutions with a mixture of Oregon Green labeled biocytin (OG) and Phycoerythrin labeled biotin (PE) (Molecular Probes, OR). The biotin-streptavidin bond, a strong and highly specific interaction used as the basis for many immunoassays, attached the fluorophores to the MagMOON [Fig. 4(a)]. The OG peak serves as a reference and at the same time illustrates the principle of a competitive assay: the OG is in competition with the PE for binding sites on the particle. Although in a traditional immunoassay excess dye would be washed away, in this experiment no washing step was performed since fluorescence from the excess dye is not modulated and can be subtracted off. A MagMOON and dye solu-
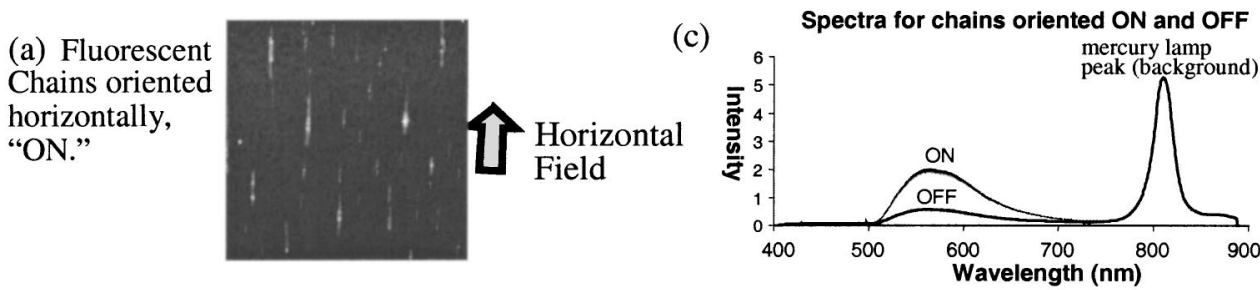

(b) Fluorescent Chains oriented into the page, "OFF."

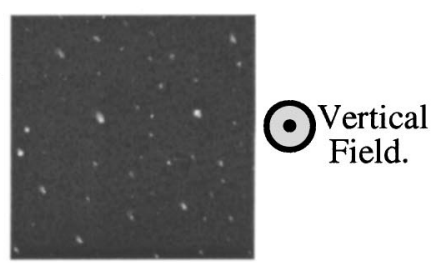

(d)

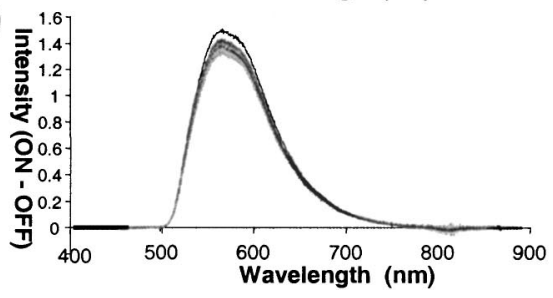

FIG. 3. Chain shaped MagMOONs. (a) Chains oriented horizontally, ON. (b) Chains oriented into the page, OFF. (c) Sixteen pairs of fluorescent spectra from chains oriented $\mathrm{ON}$ and OFF with a background peak at 800 $\mathrm{nm}$ from the mercury lamp. (d) Sixteen $(\mathrm{ON}-\mathrm{OFF})$ spectra. 
(a)

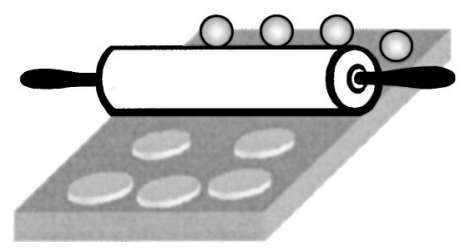

(b)

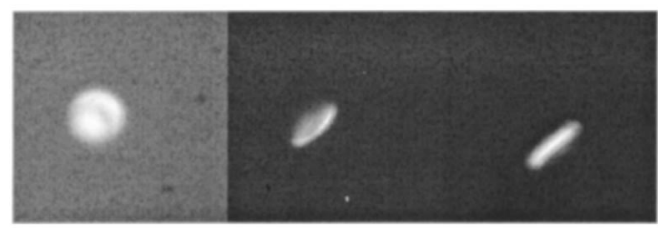

(i) "off"

(ii)

(iii) “on"

(c)

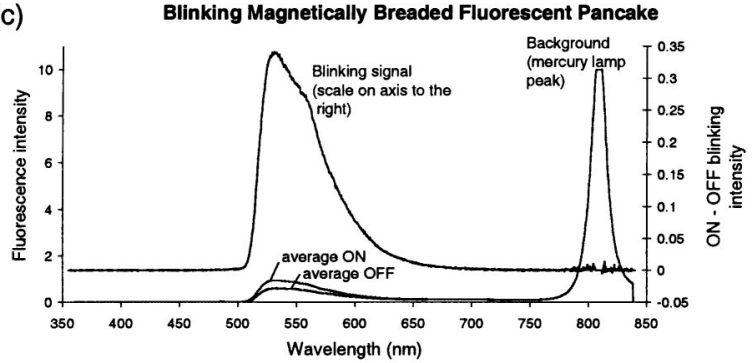

FIG. 5. Magnetically breaded fluorescent pancake. (a) Method of forming micropancakes. A rolling pin, made from a $1 / 4$ in. glass tube with a metal pin through it, is used to flatten microspheres. (b) A micropancake formed from a $3.4 \mu \mathrm{m}$ fluorescent polystyrene microsphere (Polysciences). The micropancake was breaded with magnetic material by flattening it over deposited magnetic nanoparticles; (i), (ii), and (iii) show the particle aligned in three different magnetic field orientations. Interestingly, the particle fluoresces most brightly when it is oriented edge on (rather than face on) due to total internal reflection in the particle and low self-absorption. (c) Spectral subtraction, ON minus OFF, removes background signals such as a peak at $800 \mathrm{~nm}$ from the mercury lamp.

tion was added to one well in a 96 well plate, and the solution was left overnight to let the biotin labeled dyes attach to the nanospheres. By orienting the MagMOON chains with the computer-controlled magnet, MagMOON fluorescence was separated from background fluorescence due to instrument optics, dust, and free excess biotin-labeled dyes. Thirty-two pairs of ON and OFF spectra were collected, and the average ON, OFF, and ON minus OFF spectra were plotted in Fig. 4(b). The background mercury lamp peak at 800 $\mathrm{nm}$ was attenuated by a factor of 2000 .

Chains of magnetic particles can be linked together permanently by heating the chains up above their glass transition temperature $\left(94^{\circ} \mathrm{C}\right.$ for polystyrene) in solution (e.g., within a $20 \mathrm{ml}$ vial). This process is simpler, and can yield more particles than previous methods such as: chemically linking magnetic chains, ${ }^{12}$ depositing microspheres in a groove shaped template to form chains and then melting them together in the groove,,$^{13}$ or passing microspheres through a microfluidic device where they are briefly heated. ${ }^{14}$

\section{PANCAKE AND ROLL SHAPED MagMOONs}

We developed roll and pancake shaped microparticles and breaded these with magnetic or fluorescent nano-crumbs in methods similar to cooking techniques. ${ }^{15}$ Microspheres are placed between two microscope slides, and deformed into roll (cylinder) shaped particles by moving the top surface back and forth in a reciprocal motion. Similarly, pressing a rolling pin over the particles flattens them into micropancakes (disks). Smaller particles, "nano-crumbs," can be physically embedded, "breaded," into the surface of rolls and pancakes by adding the nano-crumbs to the slide prior to rolling (Fig. 5). These processes all occur at room temperature.

In addition to improved immunoassays, MagMOONs promise to improve intracellular measurements where autofluorescence limits the range of dyes and samples that are practical. Our recently developed probe encapsulated by biologically localized embedding (PEBBLE) nanosensors measure concentrations of ions and small molecules within a single cell, rapidly, sensitively, and with high spatial resolution, without interference from cellular proteins. ${ }^{3}$ Combining them with MagMOONs will allow highly sensitive detection of intracellular analytes using a broad range of dyes. Such measurements lead to a better understanding of how cells function and how cells are effected by drugs, toxins and pathogens. Magnetic particles have been moved and rotated within cells for the past 50 years, principally to measure viscosity. ${ }^{10,16,17}$ However, none of those particles functioned as chemical sensors.

In conclusion, we have developed a general technique to magnetically modulate an optical probe signal and separate it from background fluorescence. MagMOONs promise to enhance the signal to noise ratio of a variety of biomedical applications including immunoassay, intracellular chemical sensors, cellular labels or tags, and protein folding studies.

\section{ACKNOWLEDGMENTS}

The authors thank Dr. Eric Monson for technical assistance and Dr. Martin Philbert for biological insights. The authors also acknowledge support from NSF Grant No. DMR 9900434 and NIH/NCI Contract No. N01-CO-07013.

${ }^{1}$ T. A. Byassee et al., Anal. Chem. 72, 5606 (2000).

${ }^{2}$ R. Kopelman and W. H. Tan, Science 262, 1382 (1993).

${ }^{3}$ E. Monson et al., Biomedical Photonic Handbook, edited by T. Vo-Dinh CRC, Boca Raton, FL (in press).

${ }^{4}$ R. P. Haugland, Handbook of Fluorescent Probes and Research Chemicals, 6th ed. (Molecular Probes, Inc., Eugene, OR, 1996).

${ }^{5}$ K. R. Rogers, Mol. Biotechnol. 14, 109 (2000).

${ }^{6}$ Z. Darzynkiewicz et al., Exp. Cell Res. 249, 1 (1999).

${ }^{7}$ V. Subramaniam et al., IEEE J. Sel. Top. Quantum Electron. 2, 1107 (1996)

${ }^{8}$ X. W. Zhuang et al., Science 296, 1473 (2002).

${ }^{9}$ I. Safarik and M. Safarikova, J. Chromatogr., B: Biomed. 722, 33 (1999).

${ }^{10}$ P. A. Valberg and J. P. Butler, Biophys. J. 52, 537 (1987).

${ }^{11}$ J. N. Anker and R. Kopelman, Appl. Phys. Lett. 82, 7 (2003).

${ }^{12}$ E. M. Furst et al., Langmuir 14, 7334 (1998).

${ }^{13}$ Y. D. Yin et al., J. Am. Chem. Soc. 123, 8718 (2001).

${ }^{14}$ L. Gabrielson and M. J. Folkes, J. Mater. Sci.: Mater. Electron. 36, 1 (2001).

${ }^{15}$ J. N. Anker et al., Eur. Cells Mater. 3, 95 (2002).

${ }^{16}$ F. H. C. Crick and A. F. W. Hughes, Exp. Cell Res. 1, 37 (1950).

${ }^{17}$ M. Keller et al., Rev. Sci. Instrum. 72, 3626 (2001). 